\title{
Application of Metabolomics to Reveal the Biological Mechanism of Toxicological Research
}

ISSN: 2637-8078

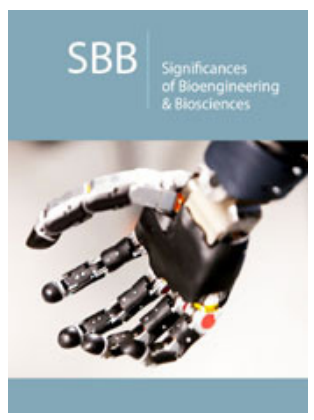

*Corresponding author: Xi Jun Wang, National Chinmedomics Research Center, National TCM Key Laboratory of Serum Pharmacochemistry, Metabolomics Laboratory, Department of Pharmaceutical Analysis, Heilongjiang University of Chinese Medicine, Harbin, China

Submission: 泟 November 09, 2020

Published: 眥December 04, 2020

Volume 4 - Issue 2

How to cite this article: Tai Ping Li, Wan Ying Wang, Hui Dong, Junling Ren, Xi Jun Wang, et al. Application of Metabolomics to Reveal the Biological Mechanism of Toxicological Research. Significances Bioeng Biosci.4(2). SBB.000583.2020. DOI: $10.31031 /$ SBB.2020.04.000583

Copyright@Xi Jun Wang, This article is distributed under the terms of the Creative Commons Attribution 4.0 International License, which permits unrestricted use and redistribution provided that the original author and source are credited.
Tai Ping Li ${ }^{1}$, Wan Ying Wang ${ }^{2}$, Hui Dong ${ }^{1}$, Junling Ren ${ }^{1}$, Hui Sun ${ }^{1}$, Fang Fang $\mathbf{W u}^{3}$, Ai Hua Zhang ${ }^{1}$ and Xi Jun Wang ${ }^{1,2,3 *}$

${ }^{1}$ National Chinmedomics Research Center, National TCM Key Laboratory of Serum Pharmacochemistry, China

${ }^{2}$ State Key Laboratory of Quality Research in Chinese Medicine, China

${ }^{3}$ National Engineering Laboratory for the Development of Southwestern Endangered Medicinal Materials, Guangxi Botanical Garden of Medicinal Plant, China

\section{Abstract}

Metabolomics can illustrate microscopic changes in the metabolic mechanisms of toxicity caused by frequent exposure to drugs, herbs, and environmental pollutants, and recent reports have supported the great potential and value of metabolomics in reducing the occurrence of injury reactions.

Keywords: Metabolomics; Toxicology; Biomarkers; Metabolic pathways; Mechanisms

Abbreviations:GC: Gas Chromatography; LC:Liquid Chromatography; MS: Mass Spectrometry; NMR:Nuclear Magnetic Resonance

\section{Introduction}

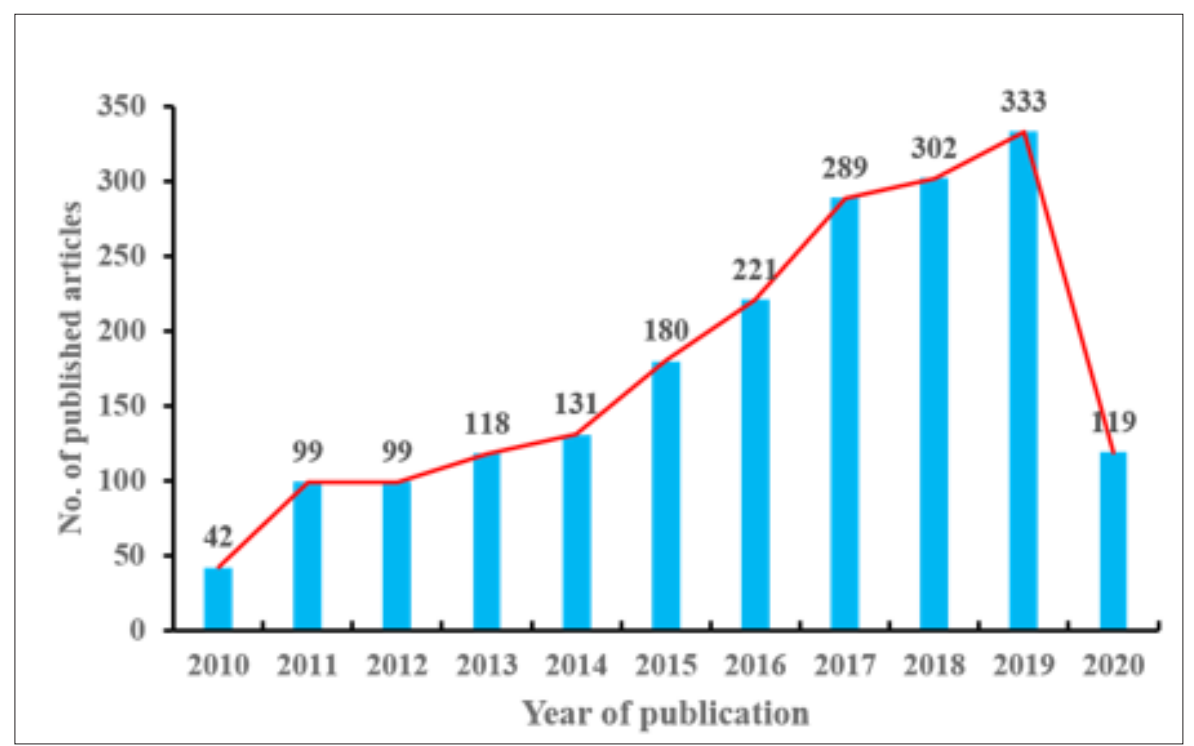

Figure 1: Histogram of metabolomics in the toxicity study manuscript, with "metabolomics, toxicity" as the key word, in their all fields were extracted from PubMed from January 2010 to September 2020. No. of publications of related articles is increasing.

Humans are often exposed to exogenoussubstances such as drugs, herbs, and environmental pollutants, which can harm health to varying degrees, and traditional toxicology regulates them more in dose and time than in mechanisms and solutions. Metabolomics practice high throughput sensitive analytical instruments to monitor metabolites with a molecular weight of less than 1000 in the organism, and mathematical models of multivariate statistical analysis find changes in metabolic contours after stimulation of organisms, verify biomarkers and metabolic mechanisms, and then take preventive, therapeutic and rehabilitation measures to 
reduce harmful effects [1,2]. Metabolomics is widely employed in toxicology studies presently (Figure 1). Their specific studies include the utilization of metabolomic methods to clarify the avoidance of biomarkers under sub-accumulation of environmental pollution, the discovery of damage metabolism mechanisms for herbal toxic target organs, in addition, regulatory drug abuse finds stable and reliable markers, including drug metabolites and biomarkers in the body, because of the transient detection of addictive drugs, which is a challenge for regulators [3-5]. In short, the future research of metabolomics in toxicology will be gradually standardized, with a wide range of applications.

High-throughput Analytical Platforms and Abundant Databases Contribute to the Study of Toxicology Mechanisms

The data matrix collected by high- throughput and highsensitivity instruments is multidimensional and detailed, in which gas chromatography (GC), liquid chromatography (LC), mass spectrometry (MS) and nuclear magnetic resonance (NMR) are the main tools of metabolomic workflow, and their respective advantages make the results of experiments complement each other in favor of the thorough clarification of the mechanism [6]. The inherent limitation of GC-MS is that it detects only volatile compounds or compounds that can be derived into volatile compounds. The high repeatability, non-destructive and noninvasive of NMR is the most important advantages of NMR in metabolomics research, NMR is mainly used for chemical identification and quantification of chemical composition of a given sample. It not limited to liquid samples, but also for solid, gas phase and tissue samples. The evolving MS technique provides a highly specific analytical tool that provides chemical information such as the exact mass of element formulas, isotope distribution patterns, structural analysis of the properties of parent and fragment ions, identification of real compound data using spectral matching, and comparison of concentration levels of different chemicals in mixed samples. MS has the advantage of analyzing secondary metabolites compared to NMR. In addition, different ion source technology may increase the number of metabolite detections. It is worth noting that there is no single analytical platform capable of fully quantifying and identifying all molecules in the sample. Therefore, in addition to 1D and 2D NMR experiments, different ionizing methods, LC-MS and GC-MS and other tandem technologies are needed to maximize the identification of different metabolites in complex samples. The electronic database contains a wealth of information on the chemical composition and biological function of the metabolites of organisms, which is conducive to accurately interpreting the activities and effects of biomarkers in metabolomics, thereby clarifying the mechanism, and performing subsequent verification [7]. It is noteworthy that the online and offline databases target preexisting metabolite information but supplementing and perfecting the database data is an important part of metabolomic progress, and it is suggested researchers should take the discovery of new bioactive compounds a major direction for metabolomic research.

\section{Application of Metabolomics Strategy to Clarify Conno-}

\section{tation of Toxic Effects}

The ideal goal of metabolomics in the research of the mechanism of toxic substances is to take targeted measures to prevent and reduce health damage. Metabolomic plans have considerable application prospects in clarifying the mechanism of biomarkers and solving the transient and difficult to detect drug abuse of new psychoactive substances, the complex composition of herbs and the unclear effective and toxic substances, and the accumulation damage of industrial chemically contaminated organisms [810]. The adverse effects they cause involve a variety of metabolic mechanisms, such as lipid metabolism affecting the cell's biofilm transport and signal recognition function, citric acid circulation interfering with normal energy metabolism and oxidative stress, and amino acid metabolism disorders causing abnormal synthesis of functional proteins. Combined with general physiological and biochemical examination, the metabolic mechanism of biomarkers can be accurately identified, and corresponding preventive, therapeutic, diagnostic and aftercare measurements can be taken to reduce the occurrence of injury response and economic loss.

\section{Discussion and Conclusion}

The application potential and value of metabolomics in toxicological research are worth developing, while challenges were still existed. Unified analytical standards and principles are the main directions for the future supplement and update of metabolomics. In addition, the results of metabolomics studies are expressed in the form of biomarkers and metabolic pathways. It is necessary to identify and clarify the metabolic changes of adverse reactions caused by herbal medicine and chemicals. It is important to establish the correlation between exogenous substances and biomarkers, which makes the corresponding preventive measures more specific and targeted [11-12]. In short, maintaining the biological sample activity of metabolomics analysis, perfecting the database, using a variety of analytical instruments, standardizing the operation process thus, to obtain a stable, reliable and reproducible result. In addition, it is believed the combination of metabolomics and other advanced omics techniques can effectively improve the quality of toxicology research in the future.

\section{References}

1. Xiong H, Zhang AH, Zhao QQ, Yan GL, Sun H et al. (2019) Discovery of quality-marker ingredients of Panax quinquefolius driven by highthroughput chinmedomics approach. Phytomedicine 74: 152928.

2. Viant MR, Ebbels TMD, Beger RD, Ekman DR, Epps DJT, et al. (2019) Use cases, best practice and reporting standards for metabolomics in regulatory toxicology. Nat Commun 10(1): 3041.

3. Geng N, Ren X, Gong Y, Zhang H, Wang F, et al. (2019) Integration of metabolomics and transcriptomics reveals short-chain chlorinated paraffin-induced hepatotoxicity in male sprague-dawley rat. Environ Int 133(Pt B): 105231.

4. Zhang Z, Yang L, Huang X, Gao Y (2020) A serum metabolomic study on rats induced by polygoni multiflori radix and polygoni multiflori radix 
preparata by pattern recognition and pathways analysis. Biol Pharm Bull 43(2): 306-318.

5. Dinis Oliveira RJ (2019) Metabolism and metabolomics of opiates: A long way of forensic implications to unravel. J Forensic Leg Med 61: 128-140.

6. Wang XJ, Ren JL, Zhang AH, Sun H, Yan et al. (2019) Novel applications of mass spectrometry-based metabolomics in herbal medicines and its active ingredients: Current evidence. Mass Spectrom Rev 38(4-5): 380402.

7. Zhang A, Sun H, Wang X (2018) Mass spectrometry-driven drug discovery for development of herbal medicine. Mass Spectrom Rev 37(3): 307-320.

8. Deng P, Barney J, Petriello MC, Morris AJ, Wahlang B, et al. (2019) Hepatic metabolomics reveals that liver injury increases PCB 126-induced oxidative stress and metabolic dysfunction. Chemosphere 217: 140-149.
9. Steuer AE, Brockbals L, Kraemer T (2019) Metabolomic strategies in biomarker research-new approach for indirect identification of drug consumption and sample manipulation in clinical and forensic toxicology? Front Chem 7: 319.

10. Thumann TA, Pferschy Wenzig EM, Aziz Kalbhenn H, Ammar RM, Rabini S, et al. (2020) Application of an in vitro digestion model to study the metabolic profile changes of an herbal extract combination by UHPLCHRMS. Phytomedicine 71: 153221.

11. Han Y, Sun H, Zhang A, Yan G, Wang XJ (2020) Chinmedomics, a new strategy for evaluating the therapeutic efficacy of herbal medicines. Pharmacol Ther 216: 107680.

12. Guo R, Luo X, Liu J, Liu L, Wang X et al. (2020) Omics strategies decipher therapeutic discoveries of traditional Chinese medicine against different diseases at multiple layers molecular-level. Pharmacol Res 152: 104627. 